\title{
Un ejemplo de rentabilidad comunicativa en la sintaxis del español: los grupos complementarios
}

\author{
An instance of language economy in Spanish syntax: \\ complement groups
}

\author{
Tomás Jiménez Juliá \\ Universidad de Santiago de Compostela, España \\ tomaseduardo.jimenez@usc.es
}

\begin{abstract}
Coordination has been described far more rigidly than its actual usage shows. This paper describes cases of coordination structures in Spanish that break with some commonly held ideas and show that the search for communicative efficiency in languages rises above rigid formal rules.
\end{abstract}

Keywords: complex coordination, rules breaking, communicative efficiency

\section{INTRODUCCIÓN}

Para trabajar en lingüística, y particularmente en gramática, necesitamos operar con categorías discretas que nos faciliten su manejo. Sabemos, sin embargo, que la realidad es continua, y que las nociones gramaticales a menudo o son demasiado laxas, e incorporan unidades que no cumplen los requisitos de la categoría, o son insuficientes para explicar algunos comportamientos. En el caso de las clases de palabras estamos muy condicionados por la tradición, y seguimos hablando de adverbios y de preposiciones e incluyendo en las primeras, y excluyendo de las segundas, unidades que deberían estar fuera y dentro, respectivamente. En el caso de las estructuras sintácticas, nuestro inventario es más reciente, pero muestra problemas similares: hay estructuras operativas que no aparecen identificadas como tales, esto es, no son 'frases', cláusulas', 'oraciones' etc. El presente trabajo tratará breve- 
mente de uno de estos casos en los que las unidades operativas dentro de una determinada estructura, concretamente, la coordinativa, no tienen un carácter estable, sino coyuntural y, sin embargo, funcionan a todos los efectos como verdaderas unidades.

\section{ALGUNOS LUGARES COMUNES EN LA DESCRIPCIÓN DE LA COORDINACIÓN}

La coordinación ha tardado en verse como una relación sintáctica, en claro contraste con la subordinación. La gramática tradicional no contemplaba nada parecido a lo que hoy conocemos como sintaxis de la oración. La sintaxis era el estudio del correcto uso de las distintas clases de palabras: sus valores, funciones, restricciones y posibilidades a la hora de formar parte de la oración. La constatación de que ciertos elementos con forma proposicional podían establecer, en bloque, la misma relación con un sustantivo que un adjetivo u otro nombre había dado lugar a la conocida teoría de las incidentales de Port Royal. Posteriormente otros autores, en la tradición hispana, señaladamente Bello (1847), ampliaron y matizaron las conclusiones de los racionalistas y sentaron las bases para una clasificación de las 'subordinadas'. La subordinación, por tanto, tuvo sus raíces en el concepto clásico de 'rección'. El término 'subordinación' se aplicó inicialmente a las estructuras de relativo $\mathrm{y}$, posteriormente, a las completivas, que eran las que podían ser analizadas a partir del 'régimen del sustantivo'.

Ahora bien, si la subordinación fue un término nacido de una sintaxis de régimen y concordancia, no sucedió lo mismo con la coordinación. En este caso, no había un concepto claro al que la sintaxis pudiera remitirse en las gramáticas clásicas ${ }^{1}$. En efecto, todo lo que en las gramáticas anteriores al siglo XX pudiera afectar a lo que hoy conocemos como estructuras coordinadas se encontraba a propósito de la conjunción. Pero la conjunción se caracterizaba, precisamente, por carecer de régimen, con lo cual la característica más destacada de los elementos unidos por ella era la de su 'independencia'. La consecuencia es que en ningún caso se habla de 'estructuras formadas por medio de una conjunción', sino de 'elementos independientes' unidos por una conjunción. A partir del siglo XIX, aunque se conservaba la visión de los miembros coordinados como 'independientes', se empezó a prestar más atención a los requisitos de las palabras o secuencias para poder coordinarse. Bello (1847 §74) apunta a algo que va a ser común en los estudios

\footnotetext{
${ }^{1}$ Naturalmente me refiero al término 'coordinación' en su acepción gramatical especializada, ya que en sentido etimológico, como "Méthodo y orden nuevamente puesto, à lo que estaba desordenado y confuso" (Academia 1726-1739: s.v.) había sido utilizado esporádicamente en las gramáticas. Por citar un caso, Gómez Hermosilla (1835) titula la parte de sintaxis de su gramática "De la coordinación de las palabras", explicando en su primer párrafo que "El término castellano coordinación significa literalmente lo que el griego sintáxis" (1835: 133).
} 
sobre coordinación: el carácter 'análogo' de los miembros coordinados. La analogía de los miembros ha sido una constante a la hora de describir los requisitos de la coordinación, si bien ha habido básicamente dos formas de entenderla: como analogía formal o como analogía funcional.

La postura formalista es mantenida por la Academia en sus primeras redacciones $^{2}$, pero no es mayoritaria en la gramática tradicional. Fue, sin embargo, bandera de los primeros modelos de la gramática chomskiana. Autores como Gleitman (1965), Lakoff \& Peters (1966), Ross (1967), Dougherty (1970-1971), entre otros muchos, estudiaron la coordinación con distintos puntos de vista, distintos intereses y distintas propuestas (si había conjuction reduction o no, qué pasaba con el gapping, cuál era su verdadera estructura sintáctica), pero en todos ellos subyacía la idea de la identidad categorial de los miembros coordinados: para coordinarse las unidades tenían que ser iguales en categoría, y si no lo eran (guapo y con dinero) era porque había algún tipo de operación que 'camuflaba' dicha identidad.

Esta concepción se opone a la funcionalista, ya implícita en Bello ${ }^{3}$ y en la mayor parte de las gramáticas tradicionales, pero sistematizada en la sintaxis pos-chomskiana por Dik (1968), para quien los requisitos para la coordinación no eran categoriales, sino funcionales. Dik inauguró un modo de entender las restricciones de la coordinación que más o menos abiertamente es aceptado por todos hoy día: la coordinación implica relación entre miembros funcionalmente equivalentes, queriendo decir con ello que las unidades realizarían una misma función si actuaran aisladas una de la otra dentro de ese mismo contexto. Con un ejemplo, si decimos que en (3) los miembros coordinados realizan la misma función es porque si actuaran como unidades aisladas, no coordinadas, serían iguales funcionalmente, según muestran (1-2):

(1) Vi $[\text { a María }]_{\text {COMPLEMENTO DIRECTO. }}$

(2) Vi [unos perritos que se acercaban a ella $]_{\text {COMPLEMENTO DIRECTO. }}$

(3) Vi [a María y unos perritos que se acercaban a ella $]_{\text {COMPLEMENTO DIRECTO. }}$

Lo cierto es que entre las cuestiones relativas a la coordinación que centran la atención de la investigación hoy en día no está este tipo de requisitos, cuya identidad funcional se ve como un axioma, salvo en trabajos centrados en los casos de asimetrías diversas entre los constituyentes (por ejemplo Johannessen 1998). El foco se centra en las relaciones semánticas que expresa la construcción, las diferencias entre subordinación y coordinación en contenido e implicaciones, aspectos formales

\footnotetext{
2 "El enlace de las palabras por medio de las conjunciones pide que aquéllas sean de una misma naturaleza; esto es, que la relación se de nombre à nombre, de verbo à verbo, de adverbio à adverbio, etc. Así, no puede decirse Petra y buena; tú y llorar..." (Academia 1880: 234).

3 "Los complementos equivalen muchas veces a los adjetivos o a los adverbios, y por consiguiente puede la conjunción enlazarlos con aquéllos o éstos (hombre honrado y de mucho juicio; una carta bien escrita pero en mal papel)" (Bello 1847: §76).
} 
de las realizaciones coordinativas o el estatus sintáctico de la conjunción, problema en el que destaca la actual consideración de su carácter nuclear, liderado por la gramática chomskiana, la llamada ConjP (o frase conjuntiva) dentro de su minimalismo estructural, destinado a reducir todas las estructuras a una de tipo endocéntrico (Munn 1987; Kayne 1994; Johanssen 1998 o, en sentido crítico, Borsley $2005)^{4}$.

\section{REQUISITOS PREVIOS VS. RESULTADO FINAL}

3.1. Las discusiones sobre las cuestiones arriba indicadas no son el objeto de estas notas, sino otro más general (o más específico, según se mire): el carácter de mecanismo comunicativo dinámico de la coordinación, frente a su visión como estructura estática. En efecto, la gramática, incluso la de perfil comunicativo, tiene la tendencia a describir los hechos de lengua como si estuvieran absolutamente fijados. En otra ocasión (Jiménez Juliá 2015) he tratado sobre el uso de la voz media en español (el uso de $s e$ ), un mecanismo que está a disposición de cualquier verbo cuando se quiere expresar una especial afección de la acción verbal sobre el sujeto: la diferencia en español entre Tomó una cerveza viendo el partido y Se tomó una cerveza viendo el partido no es referencial, sino de voz verbal (activa/media) y, en ese sentido, cualquier verbo, incluidos los copulativos, pueden adquirir un valor de afección (tu hermano se está todo el día sin hacer nada), precisamente porque la voz media, en su acepción de afección interna, se ha convertido en un mecanismo activo para expresar ciertos contenidos dentro de las posibilidades gramaticales de la lengua y de un modo muy económico. No se trata de que haya verbos pronominales y verbos no pronominales, según la terminología habitual. Todos los verbos son potencialmente pronominales, aunque haya verbos que solo pueden manifestarse en voz media y, por tanto, son 'necesariamente' pronominales, equivalentes a los llamados 'deponentes' del latín (arrepentirse / *arrepentir, quejarse / *quejar, inmiscuirse / *inmiscuir, etc.). Hacer una clasificación estática de los verbos pronominales no responde, por tanto, a la realidad de la lengua española, si no se especifica que la clasificación hace referencia a las formas usadas exclusiva o mayoritariamente en voz media, y si no se tiene en cuenta que, como aprovechamiento de las potencialidades de la lengua que es, el uso de esta voz puede diferir en distintos ámbitos de la comunidad hispanohablante.

En el caso de la coordinación, lo que la gramática ha descrito mayoritariamente de una manera un tanto rígida son los requisitos para que las unidades puedan coordinarse, con el supuesto de que las únicas fuerzas que operan en la construcción

\footnotetext{
${ }^{4}$ La visión de la conjunción asociada al segundo miembro y no equidistante ya había sido formulada anteriormente (de Groot 1949). No así su carácter nuclear.
} 
son las condiciones previas que hacen, por ejemplo, que, en principio, no resulten viables coordinaciones como las secuencias (c) siguientes:

(4a) Juan no me puede ayudar.

(4b) Sin dinero no me puede ayudar.

(4c) *Juan y sin dinero no me pueden ayudar.

(5a) He hablado del asunto.

(5b) He hablado vehementemente.

(5c) *He hablado del asunto y vehementemente.

Las razones que se aducen son de carácter funcional y previo: Juan en (4a) es 'sujeto'; sin dinero en (4b) es un modificador circunstancial. La coordinación entre ambos se considera imposible. Lo mismo podemos decir en (5) en relación con del asunto (suplemento) y vehementemente (modificador circunstancial), cuya asociación $(5 \mathrm{c})$ se considera anómala.

Este punto de vista, pese a ser considerado plenamente funcional, no lo es salvo en un sentido muy limitado. Tiene en cuenta la función de las unidades, pero solamente dentro de contextos que implícitamente se consideran 'punto de partida' para la secuencia coordinada, como las secuencia (a) y (b), lo cual nos retrotrae a la coordination reduction (Chomsky 1965; Gleitmann 1965), según la cual una coordinación era siempre el resultado de la fusión (transformación) de secuencias independientes (en la estructura profunda). Esta coordination reduction, largamente superada en sus aspectos más superficiales, subyace, sin embargo, en la consideración de los funcionalistas, desde el propio Dik (1968), de que la coordinación solo puede establecerse entre elementos de igual función, afirmación que debe entenderse bien como falsa o como tautológica (o de Pero Grullo).

(a) Es falsa si la entendemos al modo de la conjunction reduction y lo que afirmamos es que tomando la función de esos miembros de modo independiente, esto es, previa a la coordinación, solo se coordinarán si realizan la misma (ambos sujetos, ambos complementos directos etc.). Frente a esto no hay más que ver la cantidad de coordinaciones que encontramos cuyos elementos tendrían etiquetas funcionales diferentes si no fueran miembros de la coordinación: (6-8) es una muestra:

(6) Permanecieron en la sala muy callados y durante mucho tiempo.

(7) Repartió todo lo que tenía y a casi toda su familia.

(8) Eso lo puedes hacer tú y sin ayuda de nadie.

Descartado proceso alguno de omisión en estos $\operatorname{casos}^{5}$, lo que nos proporciona la coordinación es una asociación de elementos que conjuntamente aportan una información unitaria relevante al conjunto, según veremos dentro de un momento.

\footnotetext{
${ }^{5}$ La elipsis es una eliminación de elementos repetidos más que por razones de economía, con fines enfáticos: se trata de destacar lo no eliminado. En ese sentido difiere de lo que podemos llamar 'elisión',
} 
(b) La afirmación de la identidad de función de los miembros coordinados es tautológica si consideramos el valor de las unidades dentro de la construcción coordinativa. Los miembros de una coordinación son siempre unidades enlazadas paratácticamente que aportan un valor lógico de asociación, reinterpretable como otros más específicos según el contexto, pero sin dependencias unilaterales. Son unidades 'interdependientes' en el sentido de que sin ellas el valor aportado por la construcción no existiría y, en ese sentido, 'equifuncionales'.

3.2. Si la coordinación no es una estructura tan fija y homogénea, y sus miembros no están tan sujetos a requisitos previos como se suele dar por sentado ¿cómo debe describirse? O, más exactamente ¿qué es lo relevante de la estructura coordinada como tal? Aunque ahora no puedo entrar en profundidad en ello, lo que se requiere para coordinar dos elementos es, ante todo, su identidad de clase semántica. (9) y (10) son coordinaciones extrañas, pese a que formalmente son absolutamente paralelas en forma y función:

(9) (?) Ana es simpática y espeleóloga

(10) (?) Tengo mucho frío y una cuenta en el banco

pues el Predicativo de (9) une un rasgo de naturaleza con una ocupación y el Complemento directo de (10), una sensación con una posesión alienable. Una sola horma formal puede acoger muchas nociones semánticas diferentes, pero la coordinación requiere homogeneidad en este sentido. Las restricciones de la coordinación están ligadas a transgresiones básicas de identidad semántica. De acuerdo con lo anterior, la coordinación, lejos de ser una estructura estática que impone a sus miembros unas condiciones formales rígidas, debe entenderse como un mecanismo con unos fines comunicativos muy específicos $\mathrm{y}$, como tal, como una estructura que impone la rentabilidad comunicativa a restricciones formales previas. Lo que hace la coordinación es mostrar informaciones diversas que podrían situarse en niveles jerárquicos diferentes con tratamientos sustanciales igualmente distintos, pero que mediante ella se convierten en informaciones de similar relevancia con respecto a una tercera en la que se integran. Así, en (11),

(11) Permanecieron en la sala muy callados durante mucho tiempo

tenemos una jerarquización de informaciones con valores funcionales diferentes, el asociado a muy callados (predicativo de sujeto) y el asociado a durante mucho tiempo (modificador circunstancial temporal), con una representación gráfica como (11'):

que es una eliminación asistemática y con fines económicos. Las elipsis (remática, gapping...) están bien delimitadas y tienen un carácter relativamente interlingüístico, aunque las realizaciones en distintas lenguas difieren según sus rasgos tipológicos (Jiménez Juliá 1991). En los casos que nos ocupan no hay posibilidad de explicar las estructuras apelando a omisión alguna. 
$\left(11^{\prime}\right)$ Estructura copulativa

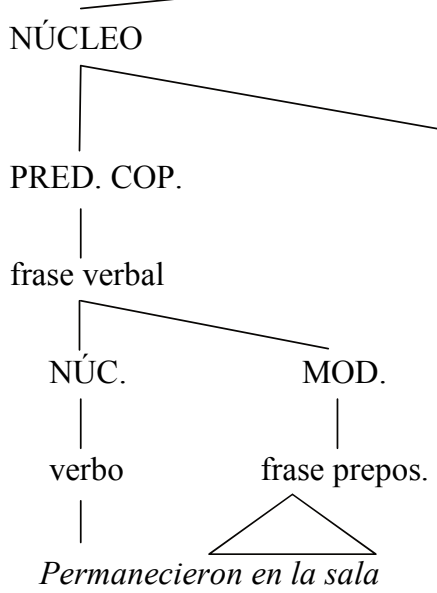

Lo que hace la coordinación es eliminar esa jerarquización y considerar que la información asociada al predicativo y la asociada al circunstancial tienen un estatus similar con respecto a una tercera. Es lo que vemos en (6), cuya representación es la indicada en (12):

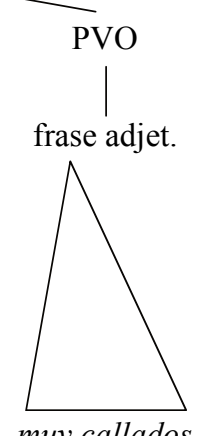

MODIFICADOR (Circ.)
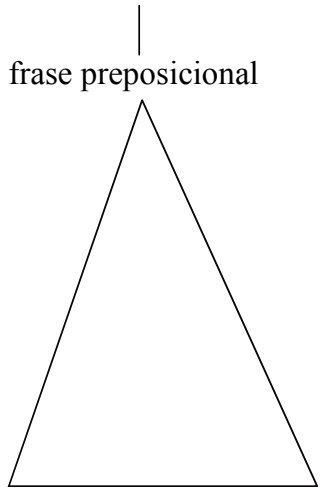

durante mucho tiempo 
Siendo la coordinación un mecanismo de neutralización de diferencias sintácticas ¿qué funciones puede neutralizar? En principio, todas. Ello explica la existencia de ejemplos como (6-8) donde las unidades coordinadas se comportan como 'Miembros' de la coordinación y, en ese sentido, y como en toda estructura paratáctica, son unidades enlazadas por un conector que establece una relación interdependiente entre ellos expresando una relación lógica (la 'conjunción' de la lógica formal) y les da un estatus funcional equivalente dentro de la construcción. $\mathrm{Y}$ ¿qué diferencia hay entre coordinaciones como las existentes en (6-8) y, por ejemplo, (13)?

(13) Me compré unos discos y unos libros

esto es, entre coordinaciones cuyo estatus funcional en secuencias independientes sería el mismo (ambos complementos 'directos'). La respuesta es: Ninguna relevante. La diferencia está en que en (13), al ser los elementos coordinados ya equivalentes en contextos no-coordinados, se hace más previsible y frecuente su coordinación, esto es, su utilización para, conjuntamente, presentarse de modo unitario con respecto a un tercero, pues si la coordinación iguala, todo aquello que ya está igualado de entrada es más susceptible de entrar en la operación coordinativa. Pero esta igualdad inicial no supone cambio alguno en lo que a la construcción resultante se refiere. Tras una unión por coordinación, el resultado final siempre será un esquema como (14):

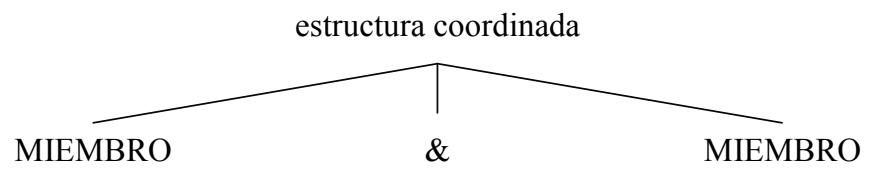

\section{GRUPOS COMPLEMENTARIOS}

Los ejemplos (6-8) muestran casos donde la lengua utiliza un mecanismo, la coordinación, para asociar unidades en principio diferenciadas $y$, de acuerdo con el grueso de la bibliografía, incoordinables, pero unidades, al fin y al cabo. La coordinación, sin embargo, es un mecanismo muy activo a la hora de equiparar informaciones con respecto a una tercera, con lo que no tiene inconveniente en traspasar, ya no las fronteras entre valores funcionales asociables a las unidades previas a su coordinación, sino incluso entre unidades como tales. En efecto, la coordinación puede operar sobre unidades que simplemente no existen en el inventario de la gramática, pero que actúan como tales en contextos específicos, esto es, en aquellos en los que la efíciencia comunicativa aconseja que así sea. Veamos los siguientes ejemplos: 
(15) Nos fuimos yo en coche y ella en tren.

(16) Compré pan para el desayuno y galletas para la cena.

(17) Juego al tenis por la mañana y al fútbol por la tarde.

Los miembros coordinados en ejemplos como (15-17), lejos de explicarse por el fácil recurso de la elipsis (nota 5 supra), son haces de constituyentes que aisladamente no constituyen unidades, pero que, asociados, actúan como tales dentro de un contexto específico donde contrastan con otros haces paralelos a través de la coordinación. Estos haces están formados por unidades cada una de las cuales conserva los rasgos sustanciales que los caracterizan como unidad funcional (sujeto, complemento directo, modificador circunstancial...), pero que se integran en un todo más amplio en el que pasan a ser miembros coyunturales de una coordinación, sin posibilidad de constituir una unidad fuera de este contexto ${ }^{6}$. Una representación estructural de (15) sería (18).

(18) frase verbal

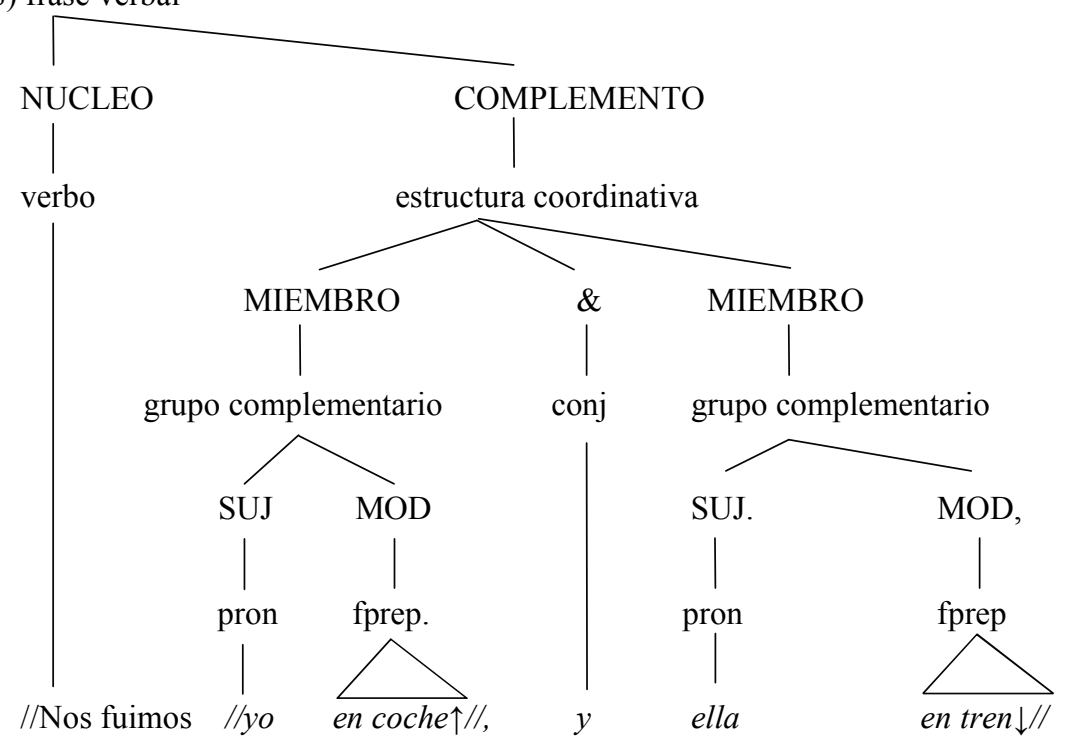

Estos grupos complementarios tienen, evidentemente, regularidades: constituyen unidades entonativas con tono continuativo la(s) primera(s), y terminativo la última, y la información compleja debe ser paralela en ambos (o más) conjuntos, pero ahondar en los detalles estructurales y prosódicos asociados a un análisis como (18)

\footnotetext{
${ }^{6}$ Los miembros de una coordinación no tienen otra relación directa que la que entablan entre ellos. Lo que puede tener relación con elementos externos es la propia construcción coordinativa. Los miembros son solo miembros de la coordinación, y han dejado de ser unidades tratables como si fueran constituyentes aislados de la construcción coordinativa.
} 
queda ya fuera de las posibilidades del presente trabajo ${ }^{7}$. Aquí simplemente quiero destacar el hecho de que este tipo de estructuras ilustra el dinamismo de las construcciones sintácticas, que no se circunscriben a la combinación de unidades utilizables en distintos contextos, como las clases de palabras y estructuras habitualmente inventariadas en las gramáticas (frases de distintos tipos, incluidas las verbales, estructuras copulativas etc.) sino que trascienden cualquier fijación cuando se dan combinaciones coyunturales que le permiten alcanzar un fin comunicativo rentable y económico. Estas líneas pretenden, sobre la base de estos ejemplos, destacar la necesidad de que la gramática describa las estructuras sintácticas en su potencialidad comunicativa y no solamente, como es habitual, en sus resultados estáticos.

\footnotetext{
${ }^{7}$ Aunque no pueda entrar en ello ahora, es preciso distinguir estas construcciones con grupos complementarios coordinados de otros como Mesas caras de caoba, y baratas de conglomerado, donde existen dos referentes (dos mesas distintas) y, a diferencia de (15-17), debemos suponer la elisión (que no elipsis) de una segunda referencia idéntica a la primera. Por ello, la interpolación aquí es natural.
} 


\section{BIBLIOGRAFÍA}

Academia Española, R. (1726-1739). Diccionario de la lengua castellana (Diccionario de Autoridades). Edición facsimil Madrid: Gredos, 1919.

Academia Española, R. (1880). Gramática de la lengua castellana. Madrid: Gregorio Hernández.

Bello, A. (1847). Gramática de la lengua castellana destinada al uso de los americanos. Edición crítica de R. Trujillo. Madrid: Arco/Libros (2 vol.), 1988.

Borsley, R.D. (2005). Against ConjP. Lingua, 115, 461-482.

Chomsky, N. (1965). Aspects of the theory of language. Cambridge, MA: The MIT Press.

Dik, S.C. (1968). Coordination. Its Implications for the Theory of General Linguistics. Amsterdam: North-Holland.

Dougherty, R.C. (1970-1971). A grammar of coordinate conjoined structures. I-II. Language, 46, 4, 850-898; 47, 2, 298-339.

Gleitman, L. (1965). Coordinating conjunctions in English. Language, 41, 260-293.

Gómez Hermosilla, J. (1835). Principios de gramática general. Madrid: Imprenta Real.

Groot, A.W. de (1949). Structurele sintaxis. The Hague.

Jiménez Juliá, T. (1991). Elipsis nominal y no-realización en español. In M. Brea y F. Fernández Rei (coord.), Homenaxe ó Profesor Constantino García, I (pp. 223-241). Santiago de Compostela: Universidade de Santiago de Compostela.

Jiménez Juliá, T. (2015). En torno a la voz media. In A. Álvarez Méndez et al. (eds.), Studium grammaticae. Homenaje al profesor José A. Martínez (pp. 489-507). Oviedo: Universidad de Oviedo.

Johannessen, J.B. (1998). Coordination. Oxford: Oxford University Press.

Kayne, R.S. (1994). The Antisymmetry of Syntax. Cambridge, MA: The MIT Press.

Lakoff, G., Peters, S. (1966). Phrasal conjunction and symmetric predicates. NSF, Cambridge Mass.

Munn, A. (1987). Coordinate structure and X-bar theory. McGill Working Papers in Linguistics, $4,1,121-140$.

Ross, J.R. (1967). Gapping and the order of constituents. In Actes du Xe Congrès International des Linguistes II, Bucarest 1970 (pp. 841-853). Bucarest. 